\title{
LIMIT DISTRIBUTION OF THE MAXIMUM AND MINIMUM OF SUCCESSIVE CUMULATIVE SUMS OF RANDOM VARIABLES
}

\section{ABRAHAM WALD}

1. Introduction. For any positive integral value $N$, let $X_{N 1}, X_{N 2}$, $\cdots, X_{N N}$ be independent and identically distributed random variables each having standard deviation 1 . Let $\mu_{N}$ denote the mean value of $X_{N i}$, and let

$$
S_{N k}=X_{N 1}+X_{N 2}+\cdots+X_{N k} .
$$

Two cases will be considered: (1) the sequence $\left\{N^{1 / 2} \mu_{N}\right\}$ converges to a finite value as $N \rightarrow \infty$; (2) $\lim _{N=\infty} N^{1 / 2} \mu_{N}=\infty$. In case (1) we shall obtain for any positive constants $a$ and $b$ the limit values of

$$
P_{N}(a)=\operatorname{prob}\left\{\max \left(S_{N 1}, \cdots, S_{N N}\right)<a N^{1 / 2}\right\}
$$

and

$$
\begin{aligned}
P_{N}^{*}(a, b)=\operatorname{prob}\left\{-b N^{1 / 2}\right. & <\min \left(S_{N 1}, \cdots, S_{N N}\right) \\
& \left.\leqq \max \left(S_{N 1}, \cdots, S_{N N}\right)<a N^{1 / 2}\right\}
\end{aligned}
$$

as $N \rightarrow \infty$. In case (2), we shall obtain for any real value $c$ the limit of

$$
Q_{N}(c)=\operatorname{prob}\left\{\max \left(S_{N 1}, \cdots, S_{N N}\right)<N \mu_{N}+c N^{1 / 2}\right\}
$$

as $N \rightarrow \infty$.

In the particular case when $\mu_{N}=0$ and $a=b$, the limit values of (1.2) and (1.3) were recently obtained by Erdös and Kac [1]. ${ }^{1}$ The case when $\mu_{N} \neq 0$, especially when $\mu_{N} N^{1 / 2}$ converges to a finite value, is of particular importance in the theory of sequential tests of statistical hypotheses. It will be seen in $\$ 3$ that the limit distribution of the number of observations required by a sequential probability ratio test can immediately be obtained from the limit values of (1.2) and (1.3), and vice versa.

2. Proof that the limit values of (1.2) and (1.3) do not depend on the distribution of the $X$ 's. It will be assumed in this section that $\mu_{N} N^{1 / 2}$ converges to a finite value as $N \rightarrow \infty$. The independence of the limit values of (1.2) and (1.3) of the distribution of the $X$ 's was proved by Erdös and Kac [1] in the special case when $\mu_{N}=0$ and $a=b$. To deal with the more general case considered here, we shall

Received by the editors, July 29, 1946.

${ }^{1}$ Numbers in brackets refer to the references cited at the end of the paper. 
follow essentially their method of proof. Let $k$ be a positive integer, and let

$$
\begin{aligned}
N_{j} & =\left[j \frac{N}{k}\right] \quad(j=1,2, \cdots, k), \\
P_{N, k}(a) & =\operatorname{prob}\left\{\max \left(S_{N N_{1}}, \cdots, S_{N N_{k}}\right)<a N^{1 / 2}\right\},
\end{aligned}
$$

and

$$
\begin{aligned}
E_{N r}=\operatorname{prob}\{ & S_{N r} \geqq a N^{1 / 2}, S_{N 1} \\
& \left.<a N^{1 / 2}, \cdots, S_{N, r-1}<a N^{1 / 2}\right\}(r=1, \cdots, N) .
\end{aligned}
$$

Let, furthermore, $\epsilon$ be a positive number. Following Erdös and Kac, for $N_{i}<r \leqq N_{i+1}$ we write

$$
\begin{aligned}
E_{N r}=\operatorname{prob}\left\{S_{N r} \geqq a N^{1 / 2}, S_{N 1}\right. & <a N^{1 / 2}, \cdots, S_{N, r-1} \\
& \left.<a N^{1 / 2},\left|S_{N N_{i+1}}-S_{N r}\right| \geqq \epsilon N^{1 / 2}\right\} \\
+\operatorname{prob}\left\{S_{N r} \geqq a N^{1 / 2}\right. & S_{N 1}<a N^{1 / 2}, \cdots, S_{N, r-1} \\
& \left.<a N^{1 / 2},\left|S_{N N_{i+1}}-S_{N r}\right|<\epsilon N^{1 / 2}\right\} .
\end{aligned}
$$

Clearly, the first of these probabilities is equal to $E_{N r}$ prob $\left\{\mid S_{N N_{i+1}}\right.$ $\left.-S_{N r} \mid \geqq \epsilon N^{1 / 2}\right\}$. Since

$$
E\left(S_{N N_{i+1}}-S_{N r}\right)^{2} \leqq\left(N_{i+1}-N_{i}\right)+\left(N_{i+1}-N_{i}\right)^{2} \mu_{N}^{2},
$$

by Tchebychef's inequality we have

$$
\begin{aligned}
\operatorname{prob}\left\{\left|S_{N N_{i+1}}-S_{N r}\right| \geqq\right. & \left.\epsilon N^{1 / 2}\right\} \\
& \leqq \frac{\left(N_{i+1}-N_{i}\right)+\left(N_{i+1}-N_{i}\right)^{2} \mu_{N}^{2}}{\epsilon^{2} N} .
\end{aligned}
$$

Since

$$
\begin{aligned}
\frac{N_{i+1}-N_{i}}{N} & =\frac{1}{N}\left\{\left[(i+1) \frac{N}{k}\right]\right\}-\frac{1}{N}\left\{\left[i \frac{N}{k}\right]\right\} \\
& \leqq \frac{1}{k}+\frac{1}{N} \leqq \frac{2}{k}
\end{aligned}
$$

we obtain from (2.6)

$$
\operatorname{prob}\left\{\left|S_{N N_{i+1}}-S_{N r}\right| \geqq \epsilon N^{1 / 2}\right\} \leqq \frac{2}{\epsilon^{2} k}\left(1+\frac{2 A}{k}\right)
$$

where $A$ is an upper bound of the sequence $\left\{N \mu_{N}^{2}\right\}$. From (2.4), (2.7) and the equation 


$$
\sum_{r=1}^{N} E_{N r}=1-P_{N}(a) \leqq 1,
$$

we easily obtain

$$
\begin{aligned}
1-P_{N}(a)= & \sum_{r=1}^{N} E_{N r} \leqq \frac{2}{\epsilon^{2} k}\left(1+\frac{2 A}{k}\right) \\
& +\sum_{i} \sum_{N_{i}<r \leqq N_{i+1}} \operatorname{prob}\left\{S_{N r} \geqq a N^{1 / 2},\right. \\
& S_{N 1}<a N^{1 / 2}, \cdots, S_{N, r-1}<a N^{1 / 2}, \\
& \left.\left|S_{N N_{i+1}}-S_{N r}\right|<\epsilon N^{1 / 2}\right\} .
\end{aligned}
$$

Clearly, the double sum is less than the probability that at least one of the sums $S_{N N_{1}}, \cdots, S_{N N_{k}}$ exceeds $(a-\epsilon) N^{1 / 2}$. Hence

$$
1-P_{N}(a) \leqq \frac{2}{\epsilon^{2} k}\left(1+\frac{2 A}{k}\right)+1-P_{N, k}(a-\epsilon) .
$$

This inequality can be written as

$$
P_{N, k}(a-\epsilon)-\frac{2}{\epsilon^{2} k}\left(1+\frac{2 \mathrm{~A}}{k}\right) \leqq P_{N}(a) .
$$

Let $G_{k 1}, \cdots, G_{k k}$ be normally and independently distributed random variables with mean $\mu / k^{1 / 2}$ and variance 1 , where $\mu=\lim _{N=\infty} N^{1 / 2} \mu_{N}$. Let, furthermore,

$$
R_{k i}=G_{k 1}+\cdots+G_{k i} \quad(i=1, \cdots, k) .
$$

It follows from the central limit theorem that

$$
\lim _{N=\infty} P_{N, k}(a)=\operatorname{prob}\left\{\max \left(R_{k 1}, \cdots, R_{k k}\right)<a k^{1 / 2}\right\} .
$$

From (2.11), (2.12) and the relation $P_{N}(a)<P_{N, k}(a)$ we obtain ${ }^{2}$

$$
\begin{aligned}
\operatorname{prob}\left\{\operatorname { m a x } \left(R_{k 1}, \cdots,\right.\right. & \left.\left.R_{k k}\right)<(a-\epsilon) k^{1 / 2}\right\}-\frac{2}{\epsilon^{2} k}\left(1+\frac{2 A}{k}\right) \\
& \leqq \liminf _{N=\infty} P_{N}(a) \leqq \limsup _{N=\infty} P_{N}(a) \\
& \leqq \operatorname{prob}\left\{\max \left(R_{k 1}, \cdots, R_{k k}\right)<a k^{1 / 2}\right\} .
\end{aligned}
$$

If the distribution of the $X$ 's is such that $\left|X_{N i}\right|$ is constant, $\lim _{N=\infty} P_{N}(a)$ exists and is a continuous function of $a$, as will be seen

2 This inequality corresponds to inequality (1) in [1]. 
in $\$ 3$. Let $P(a)$ denote this limit. Using the arguments given by Erdös and $\mathrm{Kac}$ [1, pp. 295-296], one can show that inequality (2.13) implies that for any arbitrary distribution of the $X$ 's we have

$$
\lim _{N=\infty} P_{N}(a)=P(a) \text {. }
$$

It will be seen in $\S 3$ that also $\lim _{N=\infty} P_{N}^{*}(a, b)=P^{*}(a, b)$ exists and is a continuous function of $a$ and $b$ when the $X$ 's are distributed such that $\left|X_{n i}\right|$ is constant. The proof that $\lim _{N=\infty} P_{N}^{*}(a, b)=P^{*}(a, b)$ for any arbitrary distribution of the $X$ 's can be carried out in exactly the same manner as that of (2.14).

3. Determination of the limit values of (1.2) and (1.3) when the distribution of the $X$ 's is such that $\left|X_{N i}\right|$ is constant. ${ }^{3}$ In this section it will be assumed that $\left|X_{N i}\right|$ is constant and that $N^{1 / 2} \mu_{N}$ converges to a finite value as $N \rightarrow \infty$. The limits of (1.2) and (1.3) as $N \rightarrow \infty$ can easily be obtained from some results in the theory of sequential tests of statistical hypotheses (see [2] and [3]).

The sequential probability ratio test for testing a statistical hypothesis $H_{0}$ against an alternative hypothesis $H_{1}$ is defined as follows: Let $H_{i}$ be the hypothesis that the elementary probability law of the random variable $X$ under consideration is equal to $f_{i}(x)(i=0,1)$. Let

$$
z=\log \frac{f_{1}(x)}{f_{0}(x)}
$$

and

$$
z_{k}=\log \frac{f_{1}\left(x_{k}\right)}{f_{0}\left(x_{k}\right)}
$$

where $x_{k}$ denotes the $k$ th observation on $x$. Thus, $z_{1}, z_{2}, \cdots$, ad inf.

${ }^{3}$ This problem is intimately connected with a discrete model for the Brownian motion of a particle moving in a field of constant force (that is, gravity). Let a particle starting from the origin move along the $x$-axis in such a way that in each step it can move $\Delta x$ to the right or $\Delta x$ to the left with respective probabilities $p$ and $q=1-p$. Let the duration of each step be $\Delta t$. This random walk becomes a model of the Brownian motion in a field of constant force in the limit when $\Delta x \rightarrow 0, \Delta t \rightarrow 0$, $2 p-1 \rightarrow 0$ in such a way that $(\Delta x)^{2} / 2 \Delta t=D,(2 p-1) / 2 \Delta x=c / 4 D$ where $c$ and $D$ are physical constants. The problem of finding the probability that the particle should remain in an interval around the origin during a time interval $(0, t)$ is equivalent to the problem of finding the limit value of (1.3) when $\left|X_{N i}\right|$ is constant.

The limit values of (1.2) and (1.3) are obtained here without difficulty from some previous results of the author [2]. In a subsequent publication [4], M. Kac treated the special case of a free particle $(c=0)$ using an interesting and entirely different method of attack. His method could be extended to treat also the case when $c \neq 0$. 
are independent and identically distributed random variables. The test procedure is carried out as follows: two positive constants $a$ and $b$ are chosen. At each stage of the experiment, at the $i$ th trial for each integral value $i$, the cumulative sum

$$
Z_{i}=z_{1}+z_{2}+\cdots+z_{i} \quad(i=1,2, \cdots, \text { ad inf. })
$$

is computed. Additional observations are taken as long as $-b<Z_{i}<a$. The first time that this inequality does not hold, the test procedure is terminated. Let $n$ denote the smallest integral value of $i$ for which $Z_{i}$ does not lie in the open interval $(-b, a) . H_{1}$ is accepted if $Z_{n} \geqq a$, and $H_{0}$ is accepted if $Z_{n} \leqq-b$.

It has been shown in [2] that for all points $t$ in the complex plane for which the absolute value of $\phi(t)=E\left(e^{z t}\right)$ is not less than 1, the following identity holds :

$$
E\left[e^{Z_{n} t} \phi(t)^{-n}\right]=1 .
$$

Assume now that $z$ can take only two values, $g$ and $-g(g>0)$. Let $p$ denote the probability that $z=g$. Then the expected value of $z$ is equal to

$$
\mu=g(2 p-1)
$$

and the variance of $z$ is given by

$$
\sigma^{2}=g^{2}\left[1-(2 p-1)^{2}\right]
$$

Let

$$
\begin{aligned}
m & =\frac{\mu^{2}}{2 \sigma^{2}} n, \\
t_{1}(\tau) & =\frac{1}{g} \log \frac{e^{-\left(\mu^{2} / 2 \sigma^{2}\right) \tau}-\left(e^{-\left(\mu^{2} / \sigma^{2}\right) \tau}-4 p(1-p)\right)^{1 / 2}}{2 p}
\end{aligned}
$$

and

$$
t_{2}(\tau)=\frac{1}{g} \log \frac{e^{-\left(\mu^{2} / 2 \sigma^{2}\right) \tau}+\left(e^{-\left(\mu^{2} / \sigma^{2}\right) \tau}-4 p(1-p)\right)^{1 / 2}}{2 p}
$$

where $\tau$ is a purely imaginary variable. Since the absolute value of

$$
\phi\left[t_{i}(\tau)\right]=e^{-\left(\mu^{2} / 2 \sigma^{2}\right) \tau}
$$

is equal to 1 , we may substitute $t_{i}(\tau)$ for $t$ in (3.1). We then obtain

$$
E\left(e^{Z_{n} t_{i}(\tau)} e^{m \tau}\right)=1 \quad(i=1,2) .
$$

For any random variable $u$ and any relation $R$ let $E(u \mid R)$ denote 
the conditional expected value of $u$ when $R$ holds. For any value $r$, let $[r]$ denote the smallest integer not less than $r$. Since $Z_{n}$ can take only the values $g[a / g]$ and $-g[b / g]$, equation (3.7) can be written as follows

$$
\operatorname{prob}\left\{Z_{n}=-g\left[\frac{b}{g}\right]\right\} e^{-\sigma[b / o] t_{i}(\tau)} E\left(e^{m \tau} \mid Z_{n}=-g\left[\frac{b}{g}\right]\right)
$$

$+\operatorname{prob}\left\{Z_{n}=g\left[\frac{a}{g}\right]\right\} e^{o[a / \theta] t_{i}(\tau)} E\left(e^{m \tau} \mid Z_{n}=g\left[\frac{a}{g}\right]\right)=1(i=1,2)$.

Solving the two linear equations (3.8) in the unknowns $\psi_{1}(\tau)$ $=\operatorname{prob}\left\{Z_{n}=-g[b / g]\right\} E\left(e^{m \tau} \mid Z_{n}=-g[b / g]\right)$ and $\psi_{2}(\tau)=\operatorname{prob}\left\{Z_{n}\right.$ $=g[a / g]\} E\left(e^{m \tau} \mid Z_{n}=g[a / g]\right)$, we obtain

$$
\begin{aligned}
E\left(e^{m \tau}\right) & =\psi_{1}(\tau)+\psi_{2}(\tau) \\
& =\frac{e^{\sigma[a / g] t_{1}(\tau)}+e^{-\sigma[b / o] t_{2}(\tau)}-e^{\sigma[a / g] t_{2}(\tau)}-e^{-\sigma[b / g] t_{1}(\tau)}}{e^{\sigma[a / g] t_{1}(\tau)-\sigma[b / g] t_{2}(\tau)}-e^{\sigma[a / \theta] t_{2}(\tau)-g[b / g] t_{1}(\tau)}} .
\end{aligned}
$$

We shall be interested in the limiting case when $\mu$ and $\sigma$ take a sequence of values such that

$$
\lim \mu=0, \quad \lim \sigma=0 \text { and } \lim \frac{\mu}{\sigma^{2}}=d,
$$

where $d$ is a finite value not equal to 0 . It follows from (3.2) and (3.3) that (3.10) is equivalent with

(3.11) $\quad \lim (2 p-1)=0, \quad \lim g=0$ and $\lim \frac{2 p-1}{g}=d$.

It can easily be verified that

$$
\frac{1}{g} \log \frac{e^{-\left(\mu^{2} / 2 \sigma^{2}\right) \tau}\left(1 \pm\left(1-4 p(1-p) e^{\left(\mu^{2} / \sigma^{2}\right) \tau}\right)^{1 / 2}\right)}{2 p}
$$

converges to

$$
-d\left(1 \pm(1-\tau)^{1 / 2}\right)
$$

uniformly over any finite $\tau$-interval as $\mu, \sigma$ and $\mu / \sigma^{2}$ approach the limit values given in (3.10). Hence the characteristic function of $m$ given in (3.9) converges to 


$$
\psi(\tau)=\frac{e^{-a d(1-(1-\tau) 1 / 2)}+e^{b d(1+(1-\tau) 1 / 2)}-e^{-a d(1+(1-\tau) 1 / 2)}-e^{b d(1-(1-\tau) 1 / 2)}}{e^{-a d(1-(1-\tau) 1 / 2)+b d\left(1+(1-\tau)^{1 / 2}\right)}-e^{-a d(1+(1-\tau) 1 / 2)+b d\left(1-(1-\tau)^{1 / 2}\right)}}
$$

uniformly over any finite $\tau$-interval as $\mu, \sigma$ and $\mu / \sigma^{2}$ approach the limit values given in (3.10).

The characteristic function $\psi(\tau)$ has been inverted in [2] yielding the limit distribution of $m$. Denote this limit distribution by $F(u)$, that is, $F(u)=$ prob $\{m<u\}$ where $m$ is a random variable whose characteristic function is equal to $\psi(\tau)$. The value of $F(u)$ depends on the constants $a d$ and $b d$, since these constants are involved in the characteristic function $\psi(\tau)$. To put this dependence in evidence, we shall also use the symbol $F(u \mid a d, b d)$.

We shall now express the limit value of $P_{N}^{*}(a, b)$ in terms of $F(u \mid a d, b d)$. It is assumed that $X_{N i}$ can take only two values, $g_{N}$ and $-g_{N}\left(g_{N}>0\right)$. The values $g_{N}$ and prob $\left\{X_{N i}=g_{N}\right\}$ are chosen so that the standard deviation of $X_{N i}$ is equal to 1 and the mean value of $X_{N i}$ has the prescribed value $\mu_{N}$. We consider the case when $\lim _{N=\infty} \mu_{N} N^{1 / 2}=d \neq 0$. Let the distribution of $z$ be equal to that of $X_{N i} / N^{1 / 2}$. The mean and standard deviation of $z$ are then equal to

$$
\mu=\mu_{N} / N^{1 / 2} \text { and } \sigma=1 / N^{1 / 2}
$$

respectively. Hence, the limit values of $\mu, \sigma$ and $\mu / \sigma^{2}$ as $N \rightarrow \infty$ are equal to those given in (3.10). Clearly,

$$
\begin{aligned}
P_{N}^{*}(a, b) & =\operatorname{prob}\{n>N\} \\
& =\operatorname{prob}\left\{m>\frac{\mu^{2}}{2 \sigma^{2}} N\right\}=\operatorname{prob}\left\{m>\frac{\mu_{N}^{2} N}{2}\right\}
\end{aligned}
$$

Since $\lim _{N=\infty} \mu_{N} N^{1 / 2}=d$ and since the limit distribution $F(u)$ of $m$ is a continuous function of $u$, we obtain from (3.15)

$$
\lim _{N=\infty} P_{N}^{*}(a, b)=1-F\left(\frac{d^{2}}{2} \mid a d, b d\right) \quad(d \neq 0) .
$$

The above formula is valid for $d \neq 0$. We shall now determine the limit of $P_{N}^{*}(a, b)$ when $d=0$, that is, when $\lim _{N=\infty} \mu_{N} N^{1 / 2}=0$. Since the value of $P_{N}^{*}(a, b)$ depends on the value of $d_{N}=\mu_{N} N^{1 / 2}$, we shall put this in evidence by writing $P_{N}^{*}\left(a, b \mid d_{N}\right)$.

Clearly, for any $d \neq 0$, we have

$$
\begin{aligned}
P_{N}^{*}(a+2|d|, b+2|d| \mid 0) & >P_{N}^{*}(a, b \mid d) \\
& >P_{N}^{*}(a-2|d|, b-2|d| \mid 0) .
\end{aligned}
$$


It follows from (3.17) that

$$
\begin{array}{r}
\liminf _{N=\infty} P_{N}^{*}(a+2|d|, b+2|d| \mid 0) \geqq 1-F\left(\frac{d^{2}}{2} \mid a d, b d\right) \\
\geqq \limsup _{N=\infty} P_{N}^{*}(a-2|d|, b-2|d| \mid 0) .
\end{array}
$$

If $\mu_{N}=0$, it follows from the first limit theorem of Erdös and Kac [1] that $\lim _{N=\infty} \operatorname{prob}\left\{\max \left(S_{N 1}, \cdots, S_{N N}\right)<a N^{1 / 2}\right\}$ and $\lim _{N=\infty} \operatorname{prob}\left\{\min \left(S_{N 1}, \cdots, S_{N N}\right)>-b N^{1 / 2}\right\}$ are continuous func-

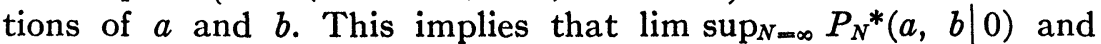
$\lim \inf _{N=\infty} P_{N}^{*}(a, b \mid 0)$ are continuous functions of $a$ and $b$, and that

$$
\begin{aligned}
& \lim _{d=0} \limsup _{N=\infty}\left[P_{N}^{*}(a+2|d|, b+2|d| \mid 0)\right. \\
&\left.-P_{N}^{*}(a-2|d|, b-2|d| \mid 0)\right]=0 .
\end{aligned}
$$

It follows from (3.18) that

$$
\begin{gathered}
\limsup _{N=\infty} P_{N}^{*}(a+2|d|, b+2|d| \mid 0) \\
-\liminf _{N=\infty} P_{N}^{*}(a+2|d|, b+2|d| \mid 0) \\
\leqq \\
\quad \lim _{N=\infty} \sup _{N}\left[P_{N}^{*}(a+2|d|, b+2|d| \mid 0)\right. \\
\left.-P_{N}^{*}(a-2|d|, b-2|d| \mid 0)\right] .
\end{gathered}
$$

Hence, because of (3.19), we have

$$
\begin{aligned}
& \lim _{d=0}\left[\limsup _{N=\infty} P_{N}^{*}(a+2|d|, b+2|d| \mid 0)\right. \\
&\left.-\lim _{N=\infty} \inf _{N}^{*}(a+2|d|, b+2|d| \mid 0)\right]=0 .
\end{aligned}
$$

The existence and continuity of $P^{*}(a, b \mid 0)=\lim _{N=\infty} P_{N}^{*}(a, b \mid 0)$ follows from the above equation and the continuity of $\lim _{\sup _{N=\infty}} P_{N}^{*}(a, b \mid 0)$ and lim $\inf _{N=\infty} P_{N}^{*}(a, b \mid 0)$. Hence, because of (3.18), we have

$$
P^{*}(a, b \mid 0)=\lim _{d=0}\left[1-F\left(\frac{d^{2}}{2} \mid a d, b d\right)\right] \text {. }
$$

Let $\left\{d_{N}\right\}$ be a sequence of values such that $\lim _{N=\infty} d_{N}=0$. Substituting $d_{N}$ for $d$ in (3.17) and letting $N \rightarrow \infty$, we obtain

$$
\lim _{N=\infty} P_{N}^{*}\left(a, b \mid d_{N}\right)=P^{*}(a, b \mid 0)=\lim _{d=0}\left[1-F\left(\frac{d^{2}}{2} \mid a d, b d\right)\right] .
$$


We shall now show that

$$
\lim _{N=\infty} P_{N}(a)=\lim _{b=\infty} \lim _{N=\infty} P_{N}^{*}(a, b) .
$$

To prove (3.22), it is sufficient to show that

$$
\lim _{b=\infty} \lim _{N=\infty} \inf \operatorname{prob}\left\{\min \left(S_{N 1}, \cdots, S_{N N}\right)>-b N^{1 / 2}\right\}=1 .
$$

Let $r$ be an upper bound of $\left|N^{1 / 2} \mu_{N}\right|$. Clearly,

$$
\begin{aligned}
& \operatorname{prob}\left\{\min \left(\bar{S}_{N 1}, \cdots, \bar{S}_{N N}\right)>(-b+r) N^{1 / 2}\right\} \\
& \leqq \operatorname{prob}\left\{\min \left(S_{N 1}, \cdots, S_{N N}\right)>-b N^{1 / 2}\right\}
\end{aligned}
$$

where $\bar{S}_{N i}=S_{N i}-i \mu_{N}$. Since according to the first limit theorem of Erdös and Kac [1] we have

$\lim _{b=\infty} \lim _{N=\infty} \operatorname{prob}\left\{\min \left(\bar{S}_{N 1}, \cdots, \bar{S}_{N N}\right)>(-b+r) N^{1 / 2}\right\}$

$$
=\lim _{b=\infty}\left(\frac{2}{\pi}\right)^{1 / 2} \int_{0}^{b-r} e^{-t^{2} / 2} d t=1
$$

(3.23) follows from (3.24). Hence (3.22) is proved.

If $\lim \left(\mu / \sigma^{2}\right)=d>0$ and $b \rightarrow \infty$, the characteristic function $\psi(\tau)$ of $m$ given in (3.13) converges to

$$
\psi^{*}(\tau)=e^{a d(1-(1-\tau) 1 / 2)} .
$$

This characteristic function has been inverted in [2] and the corresponding distribution function of $m$ is given by

$$
H(m) d m=\frac{a d}{2 \Gamma(1 / 2) m^{3 / 2}} e^{-\left(a^{2} d^{2} / 4 m\right)-m+a d} d m \quad(0 \leqq m<\infty) .
$$

Hence

$$
\lim _{N=\infty} P_{N}(a)=\operatorname{prob}\left\{m>\frac{d^{2}}{2}\right\}=\int_{d^{2} / 2}^{\infty} H(m) d m .
$$

We can summarize the results of this section in the following theorem.

ThEOREM. If $\lim _{N=\infty} \mu_{N} N^{1 / 2}=d \neq 0$, the limit value of the probability (1.3) is given by

$$
P^{*}(a, b \mid d)=\operatorname{prob}\left\{m>\frac{d^{2}}{2}\right\}
$$


where $m$ is a random variable whose characteristic function is given in (3.13). If $d=0$, the limit value of (1.3) is equal to $\lim _{d=0} P^{*}(a, b \mid d)$. For any finite value $d$, the limit value of (1.2) is equal to $\lim _{b=\infty} P^{*}(a, b \mid d)$. If $d>0$, the limit value of (1.2) is given explicitly in (3.26).

4. Derivation of the limit value of (1.4) when $\lim \mu_{N} N^{1 / 2}=\infty$. In this section we shall determine the limit value of the probability $Q_{N}(c)$ defined in (1.4) assuming that

$$
\lim \mu_{N} N^{1 / 2}=\infty \text {. }
$$

We can assume without loss of generality that $\mu_{N}>0$ for all $N$. Let $r$ be a positive number and let $\lambda(N)$ be a positive integral-valued function of $N$ such that

$$
\begin{gathered}
\lambda(N)<N, \quad \lim _{N=\infty} \frac{N-\lambda(N)}{N}=0, \\
\lim _{N=\infty} \frac{N-\lambda(N)}{N^{1 / 2}} \mu_{N}=r^{\prime}
\end{gathered}
$$

where $\infty \geqq r^{\prime}>r$. It follows from (4.1) that such a function $\lambda(N)$ exists. Because of (4.2) and (4.3) we have for sufficiently large $N$

$$
\begin{aligned}
\operatorname{prob} & \left\{\max \left(S_{N 1}, \cdots, S_{N \lambda(N)}\right)<N \mu_{N}+c N^{1 / 2}\right\} \\
& >\operatorname{prob}\left\{\max \left(S_{N 1}, \cdots, S_{N \lambda(N)}\right)<\lambda(N) \mu_{N}+(c+r) N^{1 / 2}\right\} \\
& >\operatorname{prob}\left\{\max \left(\bar{S}_{N 1}, \cdots, \bar{S}_{N \lambda(N)}\right)<(c+r) N^{1 / 2}\right\}
\end{aligned}
$$

where

$$
\bar{S}_{N i}=S_{N i}-i \mu_{N}
$$

Let $\epsilon>0$. Since for $\mu_{N}=0$ we have $\lim _{c=\infty} \lim _{N=\infty} P_{N}(c)=1$, there exists a fixed value $r_{0}$ (independent of $N$ ) such that

(4.6) $\lim _{N=\infty} \operatorname{prob}\left\{\max \left(\bar{S}_{N 1}, \cdots, \bar{S}_{N \lambda(N)}\right)<\left(c+r_{0}\right) N^{1 / 2}\right\}=1-\frac{\epsilon}{2}$.

Putting $r=r_{0}$, we obtain from (4.4) and (4.6)

(4.7) $\operatorname{prob}\left\{\max \left(S_{N 1}, \cdots, S_{N \lambda(N)}\right)<N \mu_{N}+c N^{1 / 2}\right\} \geqq 1-\epsilon$

for sufficiently large $N$. Hence

$$
\begin{aligned}
\operatorname{prob} & \left\{\max \left(S_{N 1}, \cdots, S_{N N}\right)<N \mu_{N}+c N^{1 / 2}\right\} \\
& \leqq \operatorname{prob}\left\{\max \left(S_{N, \lambda(N)+1}, \cdots, S_{N}\right)<N \mu_{N}+c N^{1 / 2}\right\} \\
& \leqq \operatorname{prob}\left\{\max \left(S_{N 1}, \cdots, S_{N N}\right)<N \mu_{N}+c N^{1 / 2}\right\}+\epsilon
\end{aligned}
$$


for sufficiently large $N$. From (4.2) and the first limit theorem of Erdös and Kac [1, p. 292] it follows easily that

$$
\begin{aligned}
\lim _{N=\infty} \operatorname{prob}\left\{\operatorname { m a x } \left(-\bar{S}_{N N}+\bar{S}_{N, N-1}, \cdots,-\bar{S}_{N N}\right.\right. & \\
& \left.\left.+\bar{S}_{N, \lambda(N)+1}\right)<\delta N^{1 / 2}\right\}=1
\end{aligned}
$$

for any positive $\delta$. Since the inequality

$$
\max \left(-\bar{S}_{N N}+\bar{S}_{N, N-1}, \cdots,-\bar{S}_{N N}+\bar{S}_{N, \lambda(N)+1}\right)<\delta N^{1 / 2}
$$

implies the validity of $\max \left(S_{N) \lambda(N,+1}, \cdots, S_{N N}\right) \leqq S_{N N}+\delta N^{1 / 2}$, we obtain from (4.9)

(4.10) $\lim _{N=\infty} \operatorname{prob}\left\{S_{N N} \leqq \max \left(S_{N, \lambda(N)+1}, \cdots, S_{N N}\right) \leqq S_{N N}+\delta N^{1 / 2}\right\}=1$

Since

(4.11) $\lim _{N=\infty} \operatorname{prob}\left\{S_{N N}<N \mu_{N}+c N^{1 / 2}\right\}=\frac{1}{(2 \pi)^{1 / 2}} \int_{-\infty}^{c} e^{-t^{2} / 2} d t$, we obtain from (4.10)

$$
\begin{aligned}
\frac{1}{(2 \pi)^{1 / 2}} & \int_{-\infty}^{c} e^{-t^{2} / 2} d t \\
& \geqq \lim _{N=\infty} \sup \operatorname{prob}\left\{\max \left(S_{N, \lambda(N)+1}, \cdots, S_{N N}\right)<N \mu_{N}+c N^{1 / 2}\right\} \\
& \geqq \liminf _{N=\infty} \operatorname{prob}\left\{\max \left(S_{N, \lambda(N)+1}, \cdots, S_{N N}\right)<N \mu_{N}+c N^{1 / 2}\right\} \\
& \geqq \frac{1}{(2 \pi)^{1 / 2}} \int_{-\infty}^{c-\delta} e^{-t^{2} / 2} d t .
\end{aligned}
$$

Since $\delta$ can be chosen arbitrarily small, it follows from (4.12) that

$$
\begin{gathered}
\lim _{N=\infty} \operatorname{prob}\left\{\max \left(S_{N, \lambda(N)+1}, \cdots, S_{N N}\right)<N \mu_{N}+c N^{1 / 2}\right\} \\
=\frac{1}{(2 \pi)^{1 / 2}} \int_{-\infty}^{c} e^{-t^{2} / 2} d t .
\end{gathered}
$$

Finally, it follows from (4.8) and (4.13), since $\epsilon$ can be chosen arbitrarily small, that

$$
\begin{gathered}
\lim _{N=\infty} \operatorname{prob}\left\{\max \left(S_{N 1}, \cdots, S_{N N}\right)<N \mu_{N}+c N^{1 / 2}\right\} \\
=\frac{1}{(2 \pi)^{1 / 2}} \int_{-\infty}^{c} e^{-t^{2} / 2} d t
\end{gathered}
$$




\section{REFERENCES}

1. P. Erdös and M. Kac, On certain limit theorems of the theory of probability. Bull. Amer. Math. Soc. vol. 52 (1946) pp. 292-302.

2. A. Wald, On cumulative sums of random variables, Ann. Math. Statist. vol. 15 (1944).

3. - Sequential tests of statistical hypotheses, Ann. Math. Statist. vol. 16 (1945).

4. M. Kac, Random walk in the presence of absorbing barriers, Ann. Math. Statist. vol. 16 (1945).

Columbia University

NOTE ON THE ZEROS OF $P_{n}^{m}(\cos \theta)$ AND $d P_{n}^{m}(\cos \theta) / d \theta$ CONSIDERED AS FUNCTIONS OF $n$

C. W. HORTON

In many physical problems in which the boundary conditions are specified over the surface of a cone, it is necessary to know the roots of the equations

$$
P_{n}^{m}(\cos \theta)=0
$$

and

$$
d P_{n}^{m}(\cos \theta) / d \theta=0
$$

considered as functions of $n$. This problem has been solved by Bholanath Pal. ${ }^{1}$ In these papers he develops infinite series for the roots $n$ which converge rapidly and are very suitable for numerical computation. In deriving his solution $\mathrm{Pal}$ introduced a parameter $k$ which takes on successive integer values and thereby yields successive roots of the equations.

It is the purpose of this note to point out that the value $k=1$ with which $\mathrm{Pal}$ commenced the series does not always give the first root of the equation, and sometimes it gives a number which is not a root of the equation. For example, in treating the equation $P_{n}^{2}(\cos \theta)=0$, $\mathrm{Pal}$ gives three roots: $n=4.77,2.26,1.52$, corresponding to values of $\theta$ equal to $15^{\circ}, 30^{\circ}, 45^{\circ}$, respectively. That these values are not roots

Received by the editors August 19, 1946.

${ }^{1}$ Bull. Calcutta Math. Soc. vol. 9 (1917-1918) p. 85; vol. 10 (1918-1919) p. 187. 